\section{Immunotherapy: A Primer for the Abdominal Radiologist}

S. Clifford, Z. Hutchinson \& A.C. O'Neill

Department of Radiology, St Vincent's Healthcare Group, Elm Park, Dublin 4, Ireland.

\section{Learning objectives}

1. Overview of names, subtypes and methods of action of immune-checkpoint inhibitors (ICI)

2. Imaging findings in immune-related

responses

3. Imaging features of immune-related adverse events (irAEs)

\section{Background}

Immune-checkpoint inhibitors, since the initial approval of ipilimumab for malignant melanoma in 2011, have demonstrated a wide spectrum of activity in various malignancies.

They have a unique method of action using the patient's immune system, which produces unusual patterns of response and toxicities.

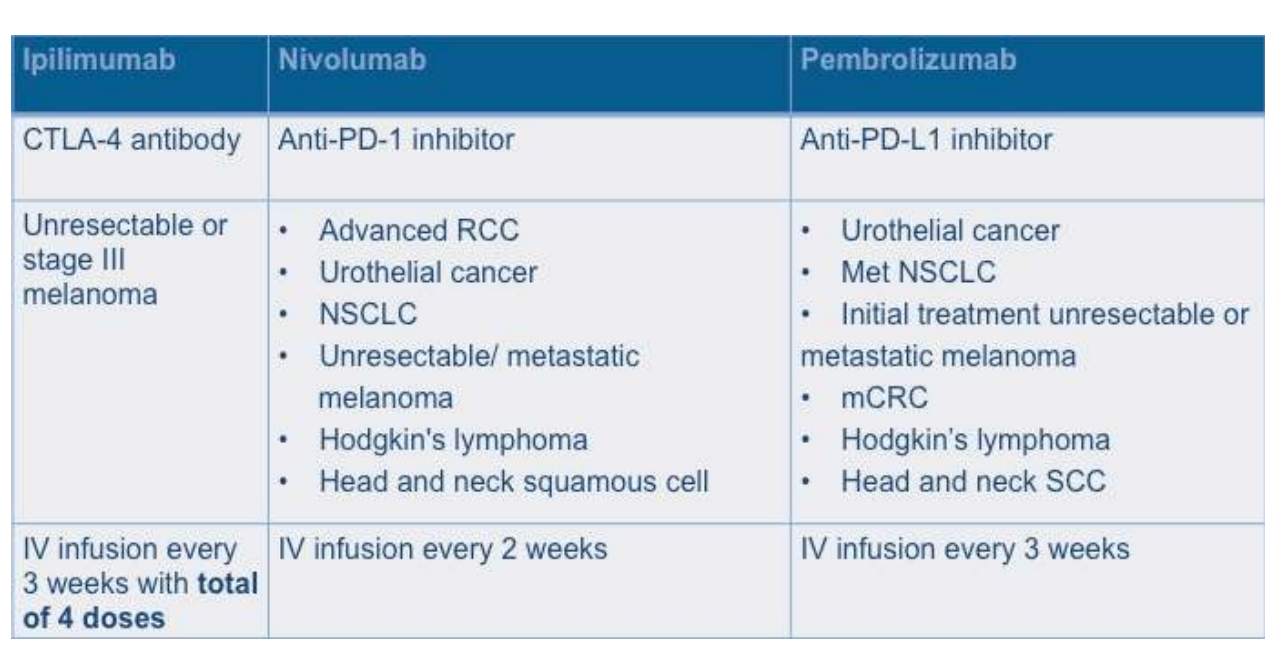

\section{Immune-related response}

Response may be seen long after the start of therapy.

Immune system modulation can be associated with inflammatory reaction causing appearance of new lesions and / or increase in size of preexisting lesions termed 'pseudoprogression'.

Immune related adverse events (irAEs)

- Colitis

- Hepatitis

- Pancreatitis

- Sarcoid-like lymphadenopathy (more commonly thoracic but can be abdominal)

Adrenalitis

- Pneumonitis (may be visible in lung bases)

- Retroperitoneal stranding
Imaging findings: Immune related response

Initial progression followed by response or stability

74 year old man with $\mathrm{mCRC}$ on nivolumab. Baseline NCCT (A) demonstrates a $128 \mathrm{~mm}$ hepatic metastasis. On initial follow up CT (B) there is enlargement of an upper abdominal ymph node. This was followed by gradual response and NCCT 2 years later shows a decrease in the hepatic metastasis $(C)$.

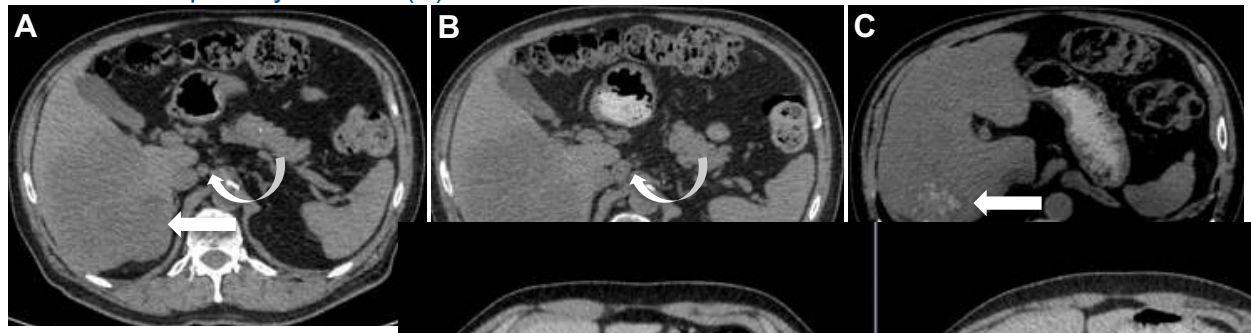

Prolonged stable disease followed by response 63 year old woman with $\mathrm{MCRC}$ on ipilimumab and nivolumab. Baseline CECT (A, B)

demonstrates partially calcified nodal metastases and a hepatic metastasis that were stable for 14 months. On follow up CECT at 18 months (C,D) there is decrease in size consistent with a partia response.

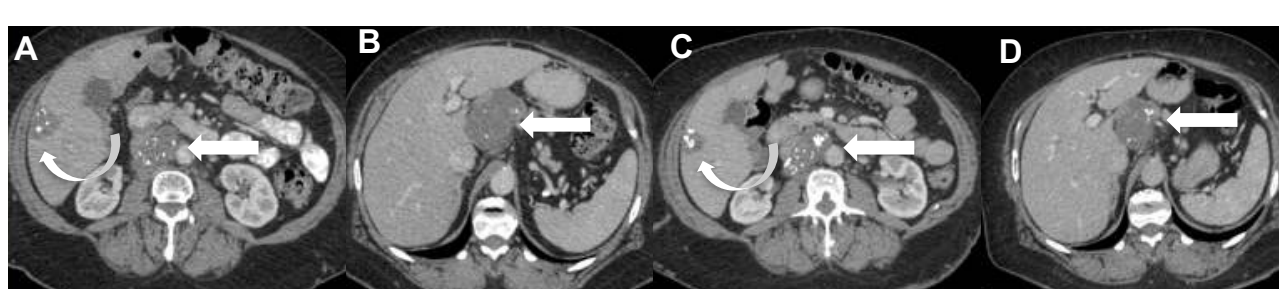

Long period of stability

51 year old woman with $\mathrm{mCRC}$ on ipilimumab and nivolumab. CECT at baseline shows a low density aortocaval lymph node $(A)$, which is stable on follow up CT two years later (C).
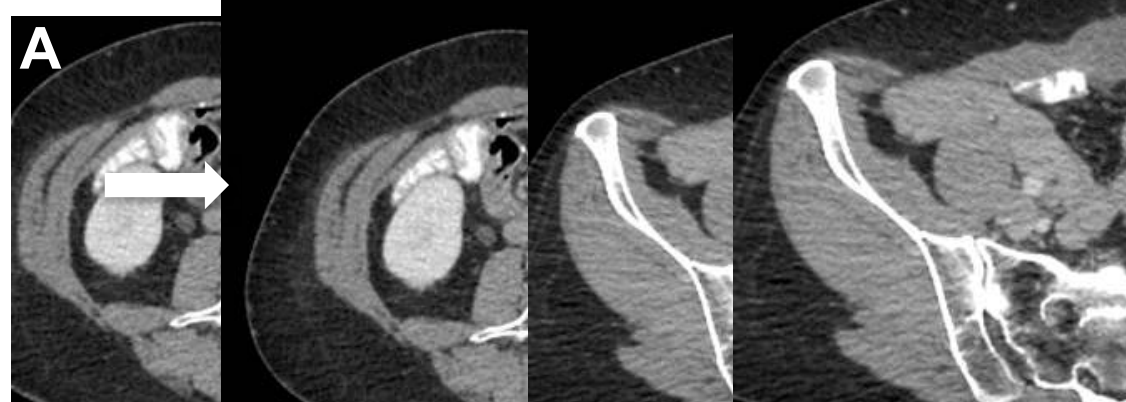
osseous metastases (D).
No new lesions and decrease in disease by 12 weeks

22 year old man with metastatic melanoma on ipilimumab and nivolumab with a partial response at 3 months followed by a complete response. Baseline CECT demonstrates splenic metastases (A) and lytic metastases in the sacrum and left ilium (B). Follow up CECT demonstrates resolution of splenic metastases (C) with interval sclerosis of the

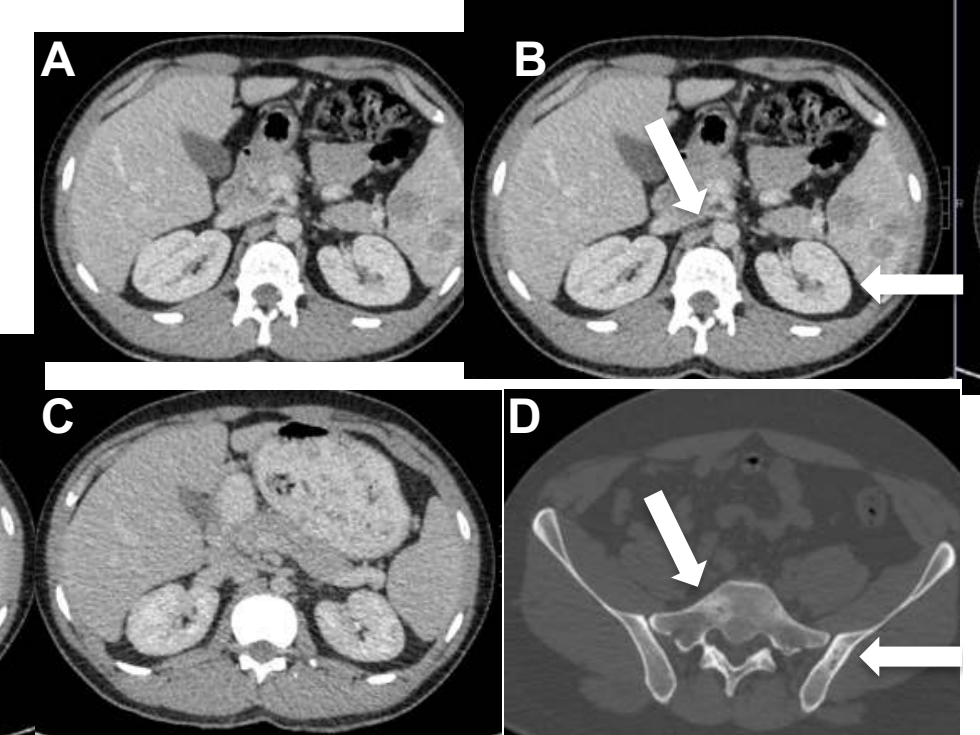

Immune related adverse events

Colitis

Most common irAE. Two different appearances

Type 1: Fluid filled colon, mesenteric vesse engorgement, mild diffuse or segmental bowel wall thickening.

Type 2: Segmental colitis associated with diverticulosis (SCAD). Segmental wall thickening with pericolonic fat stranding in pre-existing segment of diverticulosis.

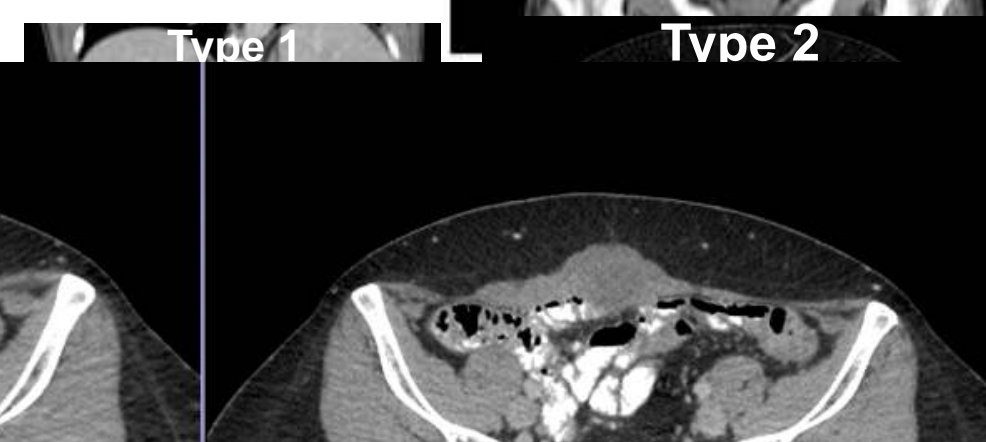

reatment of colitis

Type 1 - high dose steroids

Type 2 - high dose steroids and antibiotics

\section{Hepatitis}

Imaging findings can be subtle

- Mild hepatomegaly

- Periportal + gallbladder oedem

- Diffusely hypoattenuating or heterogeneous parenchymal enhancement

- Periportal lymphadenopathy Immune related pancreatitis

maging findings include an enlarged

pancreas with a "sausage" appearance of autoimmune pancreatitis. Can demonstrate increased FDG uptake on PET/CT.

Sarcoid like reaction

Thoracic lymphadenopathy more common but new abdominal lymphadenopathy can be seen.

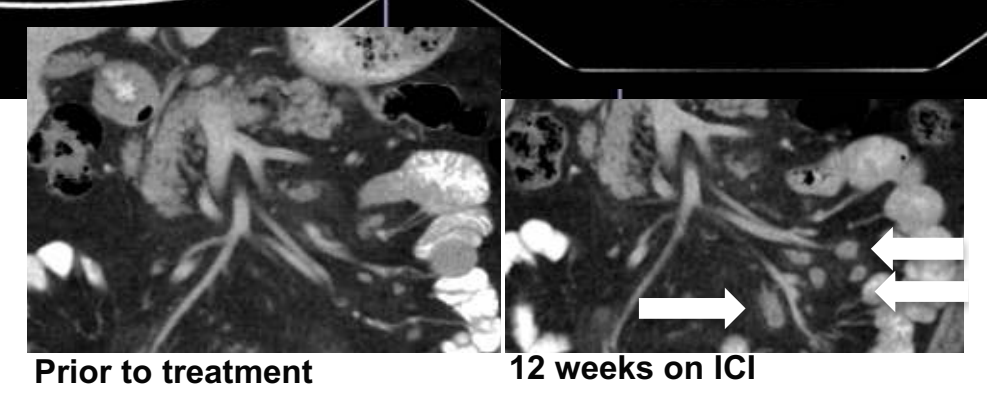

Pneumonitis

A potentially life threatening complication

Several patterns described including

- Cryptogenic organising pneumonia

- NSIP pattern

- ARDS

- Hypersensitivity pneumonia

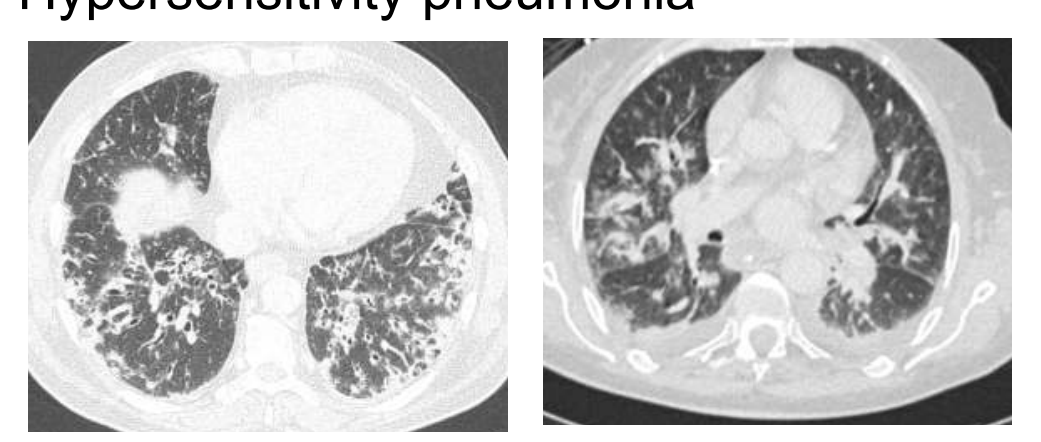

Conclusion

Immunotherapy is being increasingly used in oncology. It is important that the radiologist recognises novel immune-related patterns of response and that immune-related adverse events are recognised and reported to allow for prompt and appropriate management. 